\title{
A Role for Collapsin-1 in Olfactory and Cranial Sensory Axon Guidance
}

\author{
Hiroaki Kobayashi, Adam M. Koppel, Yuling Luo, and Jonathan A. Raper \\ Department of Neuroscience, University of Pennsylvania School of Medicine, Philadelphia, Pennsylvania 19104
}

\begin{abstract}
Collapsin-1 is a member of the semaphorin family of signaling molecules that acts as a repellent for growing spinal sensory axons. We have constructed a chimeric collapsin-1/alkaline phosphatase probe to visualize putative collapsin-1 receptors in vitro and in situ. As predicted by the activity profile of collapsin-1, the probe binds spinal sensory tracts, ventral spinal roots, and the sympathetic chain but does not bind retinal axons. In addition, we find that the probe binds sensory axons arising from the olfactory epithelium and some, but not all, cranial sensory nerves. As predicted by these binding studies, in vitro assays demonstrate that primary olfactory sensory,
\end{abstract}

trigeminal, and jugular ganglion growth cones collapse in the presence of soluble collapsin-1. Comparing the expression pattern of collapsin-1 with the trajectories of collapsin-1 responsive axons suggests that in both the spinal cord and the olfactory bulb, collapsin-1 prevents premature entry of sensory axons into their target and helps determine the final location of sensory terminations.

Key words: collapsin-1; semaphorin-III; semaphorin-D; olfactory bulb; chick hindbrain; axon guidance; alkaline phosphatase fusion protein; collapsin-1 receptors
Growth cones at the tips of extending axons must be able to detect and respond appropriately to attractive and repellent guidance cues in their immediate environment. It is likely that many of these guidance cues are signaling molecules that bind and activate specific cell surface receptors. The trajectory of a growth cone will depend on the repertoire of receptors expressed on its surface and on the effects that activation of these receptors have on its motile apparatus. Notable examples of receptors important for growth cone guidance now include unc-40 or deleted in colorectal cancer (Hedgecock et al., 1990; Serafini et al., 1994; Chan et al., 1996; Keino-Masu et al., 1996), the Eph family of receptors (Cheng et al., 1995; Henkemeyer et al., 1996; Nakamoto et al., 1996; Zhang et al., 1996), and receptor tyrosine phosphatases (Desai et al., 1996; Krueger et al., 1996).

Here we examine the embryonic distribution of binding sites, and therefore potential receptors, for the sensory axon repellent collapsin-1. Collapsin-1 is a member of a large family of signaling proteins, the semaphorins, that are expressed in specific patterns within the developing embryo (Kolodkin et al., 1993; Luo et al., 1995; Püschel et al., 1995; Adams et al., 1996). Collapsin-1 is the chick homolog of mouse semaphorin-D and human semaphorin-III. It is a secreted glycoprotein that has been shown to inhibit the motility of dorsal root ganglion (DRG) growth cones in vitro (Luo et al., 1993) and to act as a repellent of DRG axons in collagenstabilized cultures (Messersmith et al., 1995; Püschel et al., 1995). Sensory growth cones respond to concentrations of

\footnotetext{
Received May 23, 1997; revised Aug. 11, 1997; accepted Aug. 13, 1997.

This work was supported by grants from the National Institutes of Health and the McKnight Foundation. Hiroaki Kobayashi is supported by a fellowship from the Japanese Society for the Promotion of Science. We thank Dr. John Flanagan for plasmids to generate AP fusion protein and Dr. Steven Scherer for his kind advice on preparing frozen sections.

Correspondence should be addressed to Dr. Hiroaki Kobayashi, 105 Johnson Pavilion, Department of Neuroscience, University of Pennsylvania School of Medicine, 3600 Hamilton Walk, Philadelphia, PA 19104.

Copyright (C) 1997 Society for Neuroscience $0270-6474 / 97 / 178339-14 \$ 05.00 / 0$
}

soluble collapsin- 1 on the order of $10 \mathrm{pm}$ and avoid small beads to which native collapsin-1 is covalently attached (Luo et al., 1993; Fan and Raper, 1995). These findings suggest that collapsin-1 is a growth cone guidance cue that acts as a repellent and that its effects are mediated by a high-affinity cell surface receptor.

Collapsin-1 inhibits the motility of DRG, sympathetic, ciliary, and spinal motor neurons (Shepherd et al., 1996; Koppel et al., 1997) (H. Kobayashi, unpublished observations). These effects are specific, because the motilities of retinal ganglion cell and olfactory mitral cell growth cones are not inhibited by collapsin-1. Collapsin-1 mRNAs are expressed in specific, highly localized regions of the developing embryo, including the ventral spinal cord, dermamyotome, clusters of cells in the brainstem, and the olfactory bulb (Giger et al., 1996; Shepherd et al., 1996). The widespread but selective distribution of collapsin-1 mRNA in the developing embryo and the ability of several different cell types to respond to collapsin-1 indicate that it is likely to play a role in a variety of axon guidance decisions in vivo.

A systematic search for axonal guidance decisions affected by collapsin-1 would begin by identifying all the neuron types that are responsive to collapsin-1. Their axon trajectories would then be compared with the expression pattern of collapsin-1 to identify locations where collapsin- 1 is likely to act as a guidance cue. We have constructed a chimeric collapsin-1/alkaline phosphatase (AP-collapsin-1) probe that should bind and thereby allow the visualization of collapsin-1 receptors (Flanagan and Leder, 1990). We have used this probe to identify axon tracts likely to be responsive to collapsin-1. In selected cases we have confirmed that tracts that bind the probe are indeed responsive to collapsin-1 in an in vitro growth cone collapse assay. A comparison of this information with the known distribution of collapsin-1 in the embryo suggests specific instances in which this signaling molecule may play a role in growth cone guidance in vivo. This approach could provide a general method to determine system- 
A

\begin{tabular}{|c|c|c|}
\hline $\mathrm{N}$-termina & COLLAPSIN-1 & C-terminal \\
\hline (a) Col-1-AP & SEMA & $A$ \\
\hline AP & SEMA & (b) $\mathrm{AF}$ \\
\hline
\end{tabular}

B

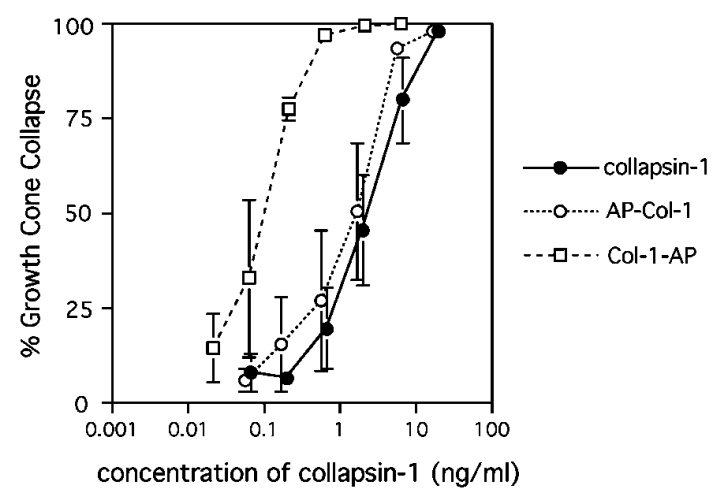

C

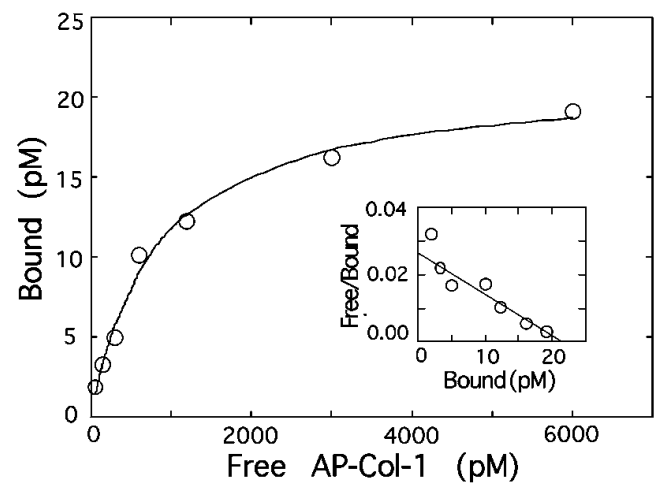

D

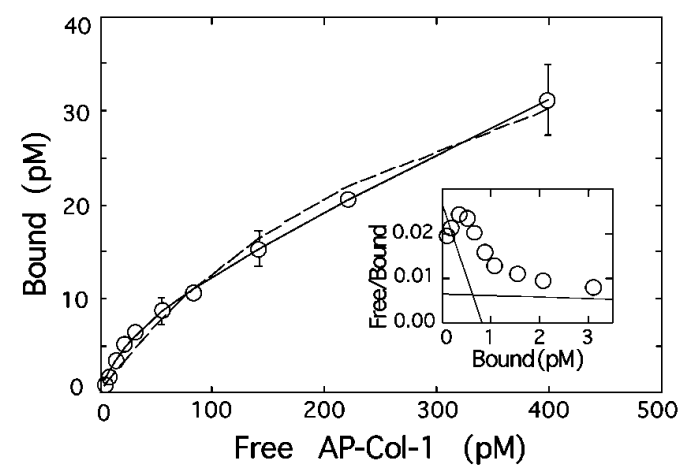

Figure 1. Characteristics of alkaline phosphatase-f used collapsin-1. A, A schematic diagram of the probes used in this study. Collapsin-1 (brackets) consists of its own signal sequence (SS), a semaphorin domain (SEMA), a C2-type immunoglobulin domain $(I g)$, and a positively charged $\mathrm{C}$ terminal tail $(++)$. $a$, The Col-1-AP probe has alkaline phosphatase $(A P)$ fused just after the basic tail of collapsin-1. $b$, The AP-Col-1 probe has a signal sequence and $A P$ fused onto the $\mathrm{N}$-terminal end of collapsin-1. $B$, The relative responsiveness of chick E7 DRG growth cones to medium conditioned by 293T cells transfected with collapsin-1 (closed circles), AP-Col-1 (open circles), and Col-1-AP (open squares). The percentages of collapsed growth cones are plotted against the log of the estimated concentrations of collapsin-1 or AP-fused collapsin- 1 . The concentrations of collapsin- 1 and AP-fused collapsin-1 in the medium were estimated by comparing the AP activities and/or the amount of atically candidate functions for other collapsin and semaphorin family members.

\section{MATERIALS AND METHODS}

Materials. The following materials were obtained from the indicated sources: nerve growth factor (NGF), brain-derived neurotrophic factor (BDNF), and neurotrophin-4 (NT4) from Alamone Labs (Jerusalem, Israel); progesterone, nitroblue tetrazolium (NBT), and 5-bromo-4chloro-3-indolyl phosphate (BCIP) from Sigma (St. Louis, MO); insulin, transferrin, and putrescine from Collaborative Research (Bedford, MA); fetal calf serum (FCS) and laminin from Life Technologies (Grand Island, NY); nitrocellulose paper from Schleicher \& Schuell (Keene, $\mathrm{NH}$ ); slot blot apparatus, Hoefer PR-648, from Hoefer Pharmacia Biotech (San Francisco, CA); EX CELL 405 from JRH Biosciences (Lenexa, KS); 1,1'-dioctadecyl-3,3,3',3'-tetramethylindocarbocyanine perchlorate (DiI) from Molecular Probes (Eugene, OR); Dispase II and alkaline phosphatase-conjugated anti-digoxigenin (DIG) antibodies from Boehringer Mannheim (Mannheim, Germany); Cy3-conjugated antibody from Jackson ImmunoResearch (West Grove, PA); Tissue-Tek OCT compound from Sakura (Tokyo, Japan); and superfrost slides from Fisher Scientific (Pittsburgh, PA).

Construction and expression of alkaline phosphatase fusion proteins. Plasmids containing alkaline phosphatase (AP) were the generous gift of Dr. John Flanagan. One contained AP with a stop codon (C-terminal fusion); the other contained AP with a leading signal sequence $(\mathrm{N}$ terminal fusion). To produce Col-1-AP, we cut out the AP sequence from the vector and inserted this sequence after the $\mathrm{C}$-terminal end of the coding sequence for collapsin-1. The amino acid sequence at the junction between collapsin-1 and AP was PRSV-(LE)-IIPV, with PRSV in collapsin-1. The two amino acids in parenthesis served as a linker. To produce AP-Col-1, we replaced the signal sequence of collapsin-1 with the AP signal and coding sequences. The amino acid sequence at the junction between AP and collapsin-1 was SGRS-(GS)-KNNV, with KNNV in collapsin-1. Recombinant protein was produced using the expression plasmid pAG3, derived from a pcDNA3 backbone (Invitrogen, San Diego, CA) with a cytomegalovirus enhancer, a chick $\beta$-actin promoter, a rabbit $\beta$-globin splice site, a bovine growth hormone polyadenylation site, and a hygromycin resistance gene (Miyazaki et al., 1989).

These two constructs, Col-1-AP and AP-Col-1, were transfected into transformed human kidney epithelial cells (293T) using calcium phosphate precipitation. Chloroquine (1:1000 of $25 \mathrm{~mm}$ stock solution) was added to the cells at the time of transfection. After a 4-5 hr incubation at $37^{\circ} \mathrm{C}$, the cells were washed and cultured in DMEM with $10 \%$ heat-inactivated FBS. Conditioned media containing the secreted fusion proteins were collected $\sim 20 \mathrm{hr}$ later.

Determining AP-collapsin-1 concentrations. To determine the relative amounts of AP-collapsin-1 fusion proteins produced in each transfection, we blotted a dilution series of culture supernatants onto nitrocellulose paper with a Hoefer slot blot apparatus and reacted the series for AP activity. Supernatants were diluted with DMEM containing $10 \%$ heatinactivated FCS. The nitrocellulose paper was rinsed in PBS and heatinactivated at $65^{\circ} \mathrm{C}$ for $3 \mathrm{hr}$ in PBS. The paper was then reacted with an AP reaction mixture containing $100 \mathrm{~mm}$ Tris, pH 9.5, $100 \mathrm{~mm} \mathrm{NaCl}, 5$ $\mathrm{mM} \mathrm{MgCl}_{2}, 0.33 \mathrm{mg} / \mathrm{ml} \mathrm{NBT}$, and $0.17 \mathrm{mg} / \mathrm{ml} \mathrm{BCIP}$. To compare the relative amounts of AP-collapsin-1 and standardized unlabeled collapsin-1 of known potency, we probed slot blots with monoclonal

collapsin-1 binding by mAb E7 (see Materials and Methods). $C$, Equilibrium binding of AP-Col-1 to dissociated sympathetic neurons. Sympathetic neurons were incubated with various concentrations of AP-Col-1 at $37^{\circ} \mathrm{C}$ for $1 \mathrm{hr}$. The cells were then fixed, washed, and assayed colorimetrically for bound AP activity. The AP-Col-1 binding to sympathetic neurons is saturable, and the $K_{\mathrm{d}}$ is estimated as $800 \mathrm{pM}$. Inset, Scatchard plot from the same data. The data are well fitted with a straight line. $D$, Binding kinetics of AP-Col-1 to dissociated sympathetic neurons at lower concentrations of the ligand. Data points were fitted with either a two or one binding-site model by nonlinear regression analysis. Data points are better fitted with the two (solid line) than with the one (dashed line) binding-site model. The $K_{\mathrm{d}}$ for the high affinity sites is $30 \mathrm{pM}$. Inset, Scatchard plot from the same data showing the presence of two binding sites. Similar results were seen in six of seven independent experiments. 

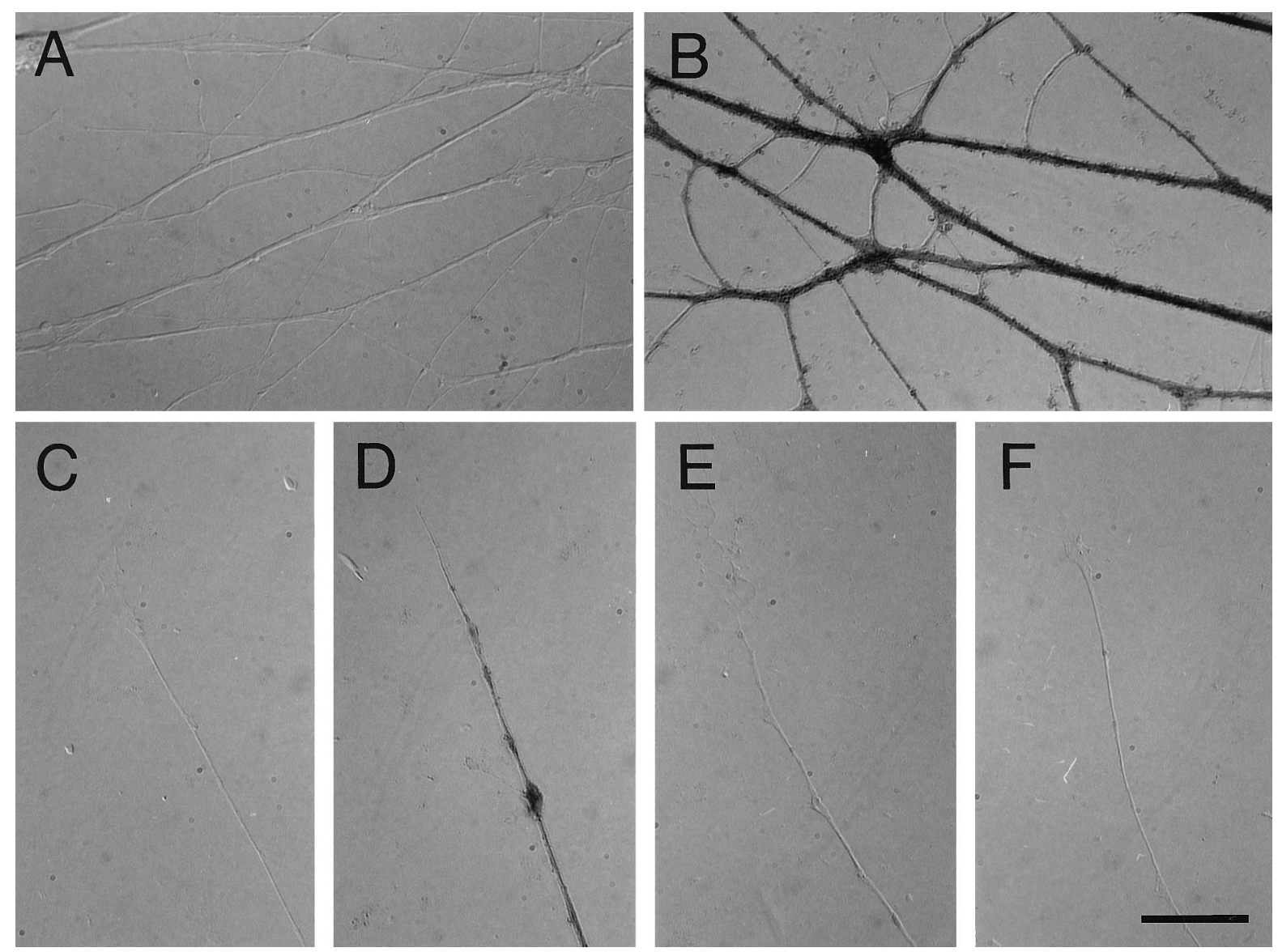

Figure 2. Col-1-AP labels DRGs but not retinal axons in vitro. Explanted E7 DRGs or fragments of E6 retina were incubated with Col-1-AP, fixed, and stained for bound AP activity. $A$, DRG axons in the absence of probe do not generate an AP reaction product. $B$, DRG axons exposed to $50 \mathrm{CU}$ of Col-1-AP stain strongly. $C$, A control DRG growth cone has no endogenous AP activity. $D$, A DRG growth cone exposed to $50 \mathrm{CU}$ of Col-1-AP is collapsed and strongly stained to its tip. E, DRG axons simultaneously exposed to $50 \mathrm{CU}$ of Col-1-AP and a 200 -fold excess of unlabeled collapsin-1 do not stain. $F$, Retinal axons treated with $50 \mathrm{CU}$ of Col-1-AP do not stain. Scale bar, $20 \mu \mathrm{m}$.

antibody (mAb) E7 anti-collapsin-1 and detected them with a HRPconjugated secondary antibody (Jackson ImmunoResearch, West Grove, PA). Estimates of relative collapsin-1 and AP-collapsin-1 concentrations were accurate to approximately a factor of two when the relative intensities of the dilution series were matched by eye.

Col-1-AP binding to cultures. DRGs from E7 chick embryos or retinae from E6 embryos were cultured in $0.5 \mathrm{ml}$ of medium on glass coverslips coated with laminin as described under Cell culture. One to $10 \mu \mathrm{l}$ of 293T cell culture supernatant containing Col-1-AP were then added to each explant culture and incubated for $1 \mathrm{hr}$ at $37^{\circ} \mathrm{C}$. The culture was fixed by the addition of $4 \%$ paraformaldehyde, $10 \%$ sucrose, and PBS. After 30 min, the culture was washed with PBS several times, the endogenous alkaline phosphatase was heat-inactivated at $65^{\circ} \mathrm{C}$ for $2 \mathrm{hr}$, and the culture was processed for AP reaction. For experiments in which unlabeled collapsin-1 was used to compete with Col-1-AP binding, $10 \mu \mathrm{l}$ of Col-1-AP containing supernatant was mixed with $10 \mu \mathrm{l}$ (containing a 20-200-fold greater concentration) of unlabeled collapsin-1 produced in a baculovirus high-expression system. The mixture was added to explant cultures and processed as described above.

Quantitative estimation of AP-Col-1 binding to cultured sympathetic cells. Approximately $5 \times 10^{4}$ dissociated sympathetic neurons were plated into each well in a 48 well plate and cultured overnight. Then AP-Col-1 was added to each well at a series of protein concentrations ranging from 8 pm to $0.4 \mathrm{~nm}$, and the cultures were incubated at $37^{\circ} \mathrm{C}$ for $1 \mathrm{hr}$. The cultures were then fixed in $4 \%$ paraformaldehyde for $30 \mathrm{~min}$, washed three times with a buffer of $0.15 \mathrm{M} \mathrm{NaCl}$ and $20 \mathrm{mM} \mathrm{HEPES,} \mathrm{pH} \mathrm{7.4,} \mathrm{and}$ incubated at $65^{\circ} \mathrm{C}$ for $2.5 \mathrm{hr}$ to destroy the endogenous alkaline phosphatase activity. The AP-Col-1 binding activity was determined colorimetrically by measuring optical density 415 after incubation in $200 \mu \mathrm{l}$ of $1 \mathrm{~m}$ diethanolamine, $\mathrm{pH} 9.8,0.5 \mathrm{~mm} \mathrm{MgCl}_{2}, 10 \mathrm{~mm}$ L-homoarginine, 0.5 $\mathrm{mg} / \mathrm{ml} \mathrm{BSA}$, and $12 \mathrm{~mm} p$-nitrophenyl phosphate for 6-10 hr. Nonspecific binding was determined through the addition of a 100 -fold excess of recombinant collapsin-1 with AP-Col-1 and was found to be $<5 \%$ of the total binding activity. The binding data were analyzed either by the Prism II ligand analysis program or by Sigma plot.

Col-1-AP binding to sections. Chick embryos were decapitated, embedded in Tissue-Tek OCT compound, and frozen in a dry ice-acetone bath. Frozen sections were cut with a cryostat and collected on Superfrost Plus slides. Care was taken not to allow the sections to dry out. After being picked up, they were allowed to attach to the slides in a moist chamber for 2-3 min. They were then immediately frozen by putting the slides onto a precooled metal stage in the cryostat. Sections were postfixed within $1 \mathrm{hr}$ of being cut with precooled methanol at $-20^{\circ} \mathrm{C}$ for $7-10$ $\mathrm{min}$. They were then washed with PBS for $5 \mathrm{~min}$ twice and blocked with PBS containing $10 \%$ FBS for $15 \mathrm{~min}$ at $20^{\circ} \mathrm{C}$. Sections were incubated with diluted $293 \mathrm{~T}$ cell culture supernatants containing Col-1-AP at $20^{\circ} \mathrm{C}$ for $1 \mathrm{hr}$. Depending on the efficiency of transfection, 293T cell culture supernatants contained 2000-5000 collapsing units $(\mathrm{CU}) / \mathrm{ml}$, and dilutions between 1:20 and 1:100 were used. Sections were rinsed with PBS; fixed with $60 \%$ acetone, $3 \%$ paraformaldehyde, and 20 mM HEPES, $\mathrm{pH}$ 7.0, for $3 \mathrm{~min}$; and then washed with PBS several times. Sections were then incubated at $65^{\circ} \mathrm{C}$ for $3 \mathrm{hr}$ to inactivate endogenous alkaline phosphatases. Sections were then processed for AP in $100 \mathrm{~mm}$ Tris, $\mathrm{pH} 9.5$, $100 \mathrm{~mm} \mathrm{NaCl}, 5 \mathrm{mM} \mathrm{MgCl} 2,0.33 \mathrm{mg} / \mathrm{ml} \mathrm{NBT}$, and $0.17 \mathrm{mg} / \mathrm{ml} \mathrm{BCIP}$ at $20^{\circ} \mathrm{C}$ overnight. For experiments in which unlabeled collapsin- 1 competed with Col-1-AP binding, collapsin-1 obtained from either a baculovirus expression system or 293T cells was used. Sections were prepared in the same way as described above and first preincubated with unlabeled collapsin- 1 for 15 min and then incubated with the mixture of the probe and unlabeled collapsin- 1 for $1 \mathrm{hr}$ and processed the same way. 

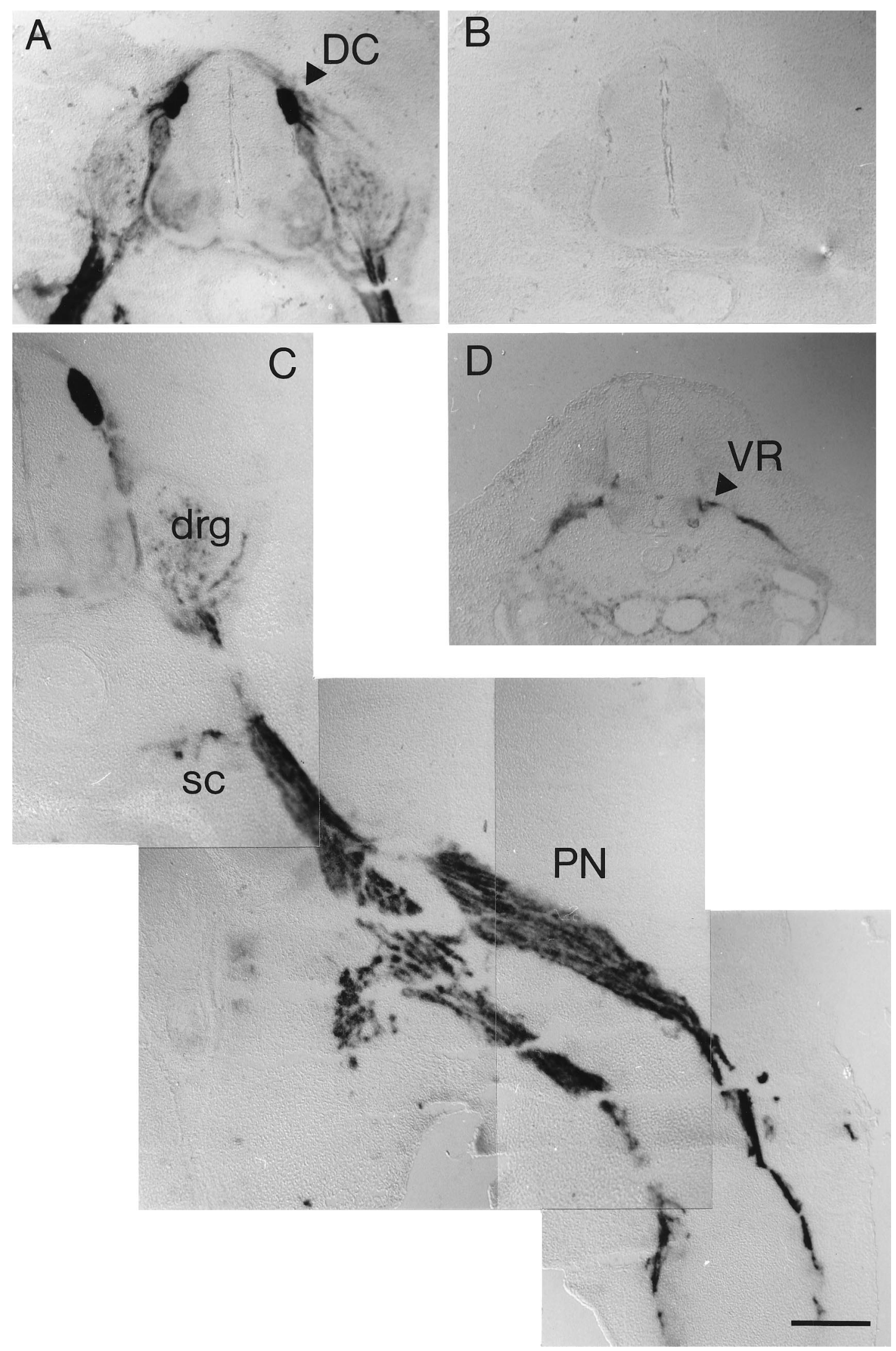

Figure 3. AP-Col-1 labels central and peripheral DRG projections in sections. $A$, The dorsal sensory columns $(D C$, arrowhead) within the spinal cord are intensely labeled by $20 \mathrm{CU}$ of AP-Col-1 in transverse sections of a stage 26 embryo spinal cord. $B$, A section from the same embryo simultaneously exposed to AP-Col-1 and an $\sim 200$-fold excess of unlabeled collapsin-1 is unstained. $C$, The peripheral nerve $(P N)$ and the sympathetic chain $(S C)$ are intensely labeled by AP-Col-1 in a section through the leg of a stage 27 embryo. $d r g$, Dorsal root ganglion. $D$, the ventral root $(V R)$ is stained with AP-Col-1 in a section through a stage 21 spinal cord. Scale bar, $200 \mu \mathrm{m}$. 

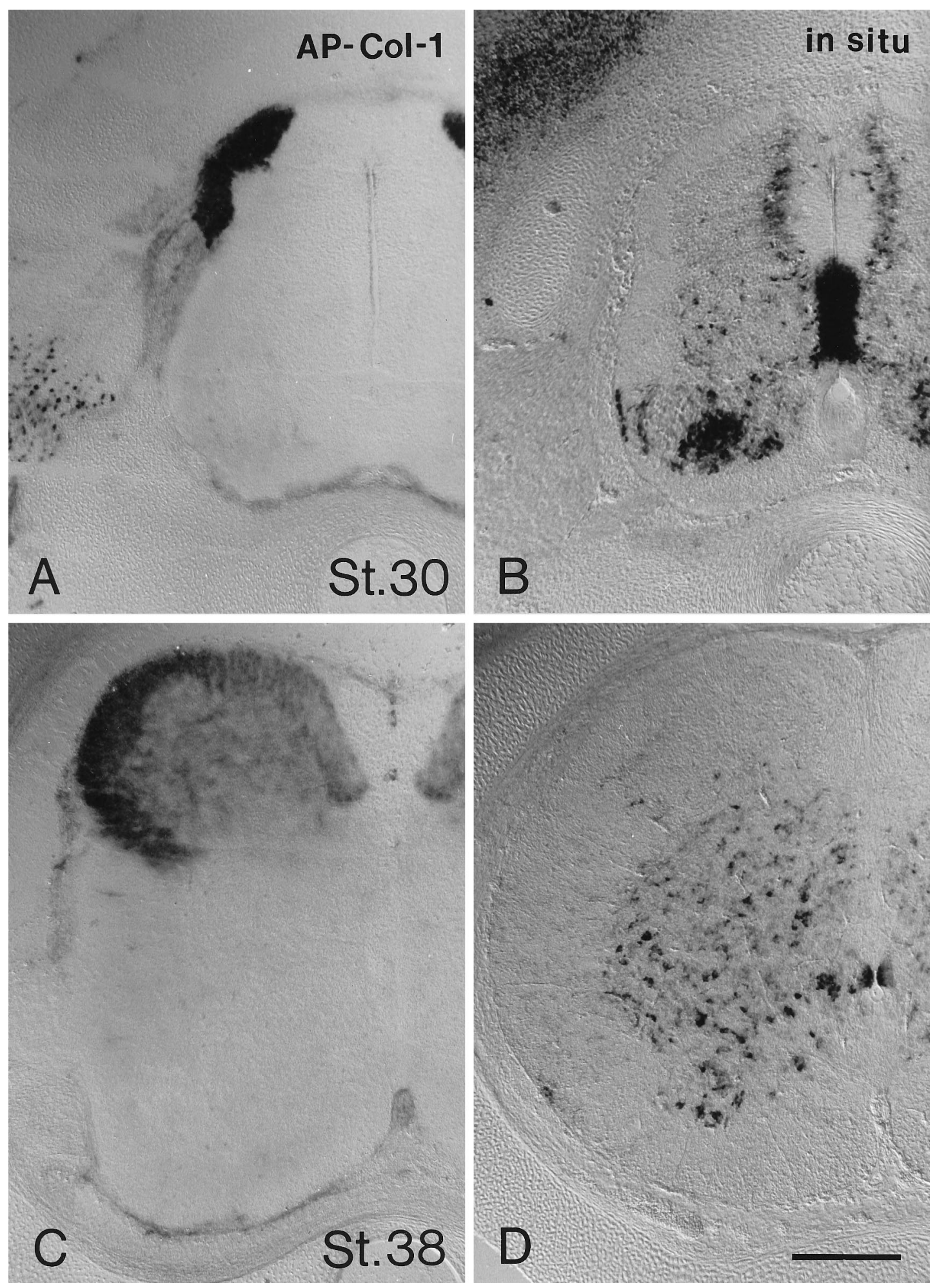

Figure 4. Two classes of sensory afferents are differentially labeled in the developing spinal cord. A, AP-Col-1 binds to dorsal column sensory tracts in a transverse section of stage 30 lumbar spinal cord. $B$, An in situ hybridization for collapsin- 1 mRNA demonstrates its medial and ventral distribution in a stage 30 spinal cord. $C$, AP-Col- 1 binds to the dorsal columns and dorsally terminating sensory afferents of a stage 38 spinal cord. There is no detectable labeling of ventrally terminating sensory afferents. $D$, An in situ hybridization for collapsin-1 mRNA demonstrates its ventral distribution in a stage 38 spinal cord. Different specimens were used for AP-Col-1 staining and for in situ hybridizations because the two techniques are incompatible. Scale bar, $200 \mu \mathrm{m}$. 

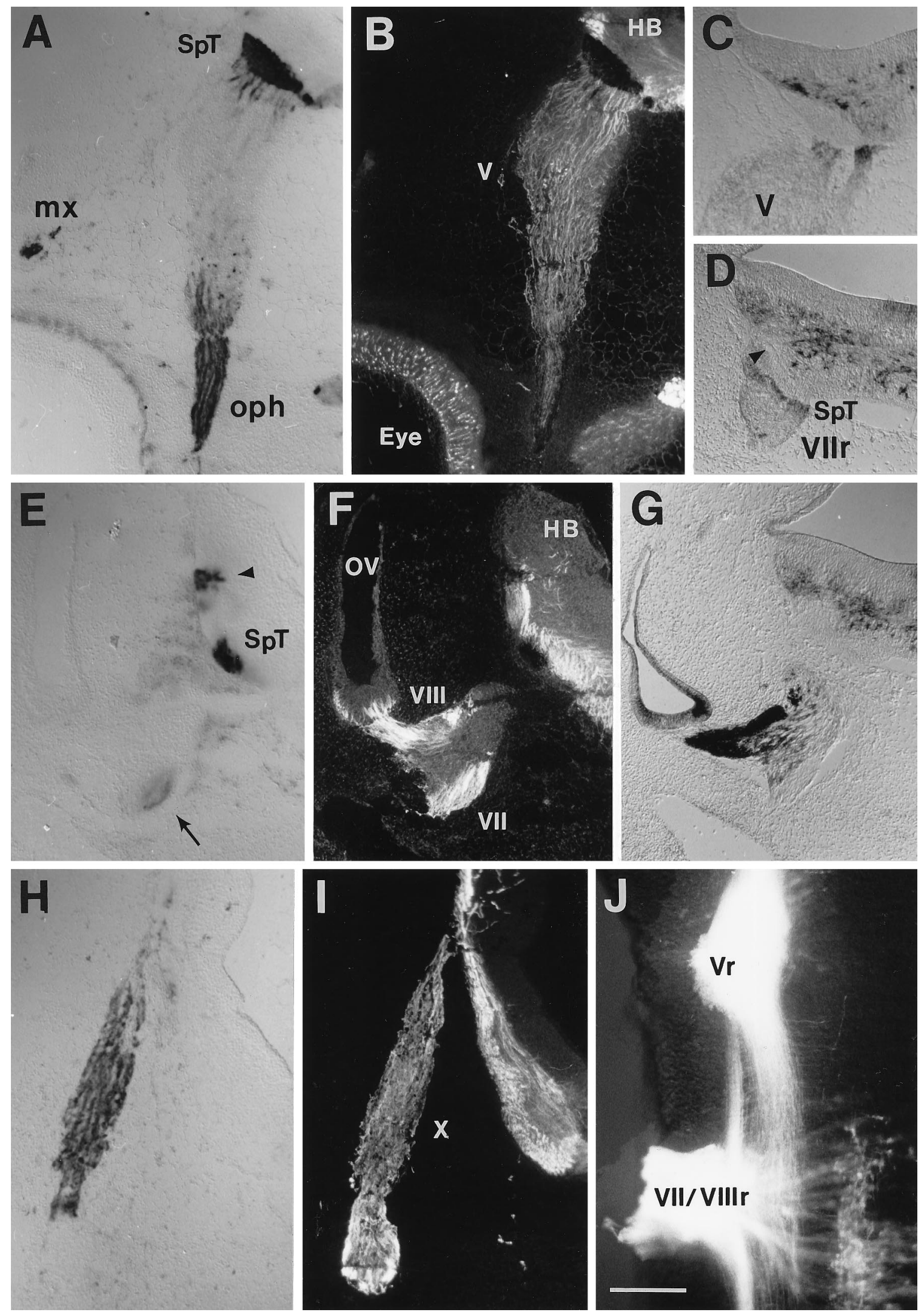

Figure 5. AP-Col-1 labeling in the cranial sensory tracts in the hindbrain. $A, \mathrm{AP}-\mathrm{Col}-1$ labels axons exiting the $\mathrm{Vth}$ (trigeminal) ganglion in a transverse section of stage 25 embryo hindbrain. The ophthalmic $(o p h)$ and maxillary nerves $(m x)$ are labeled in the periphery, and the descending trigeminal tract $(S p T)$ is labeled within the hindbrain $(H B)$. B. The same section probed with an antibody to neurofilament shows the location of other axonal tracts unlabeled with AP-Col-1. The neurofilament staining is masked by the AP reaction product where AP-Col-1 is bound. Eye, Eye; $V$, trigeminal ganglion. $C$, An in situ hybridization for collapsin-1 mRNA at the entry point for the Vth nerve $(V)$ into the hindbrain (Figure legend continues) 
When double-stained for neurofilament and AP-Col-1, sections were prepared in the same way but were first blocked with PBS containing $10 \%$ FBS and then were incubated with a mixture of hybridoma culture supernatant (4H6, anti-neurofilament) and AP-Col-1. The sections were then fixed, washed, heat-inactivated, and incubated with $\mathrm{Cy} 3$-conjugated secondary antibody for $2 \mathrm{hr}$. Sections were then fixed again, washed with PBS, and processed for AP.

Reconstruction. Images of tissue sections were taken through a television camera (Hamamatsu; C2400) attached to an inverted Zeiss microscope. Images were then projected onto a television monitor screen (Sony, Tokyo, Japan; PVM-122) and traced onto transparent sheets. These tracings were scanned into Adobe photoshop. Schematics were based on reconstructions generated by this method.

In situ hybridization. In situ hybridizations were performed as described previously with only minor variations (Shepherd et al., 1996). Sections were cut at $30 \mu \mathrm{m}$, and prehybridizations were performed for 1 $\mathrm{hr}$ at $65^{\circ} \mathrm{C}$. Alkaline phosphatase-conjugated anti-DIG antibodies were used at a 1:2500 dilution. The AP reaction was performed as described under Col-1-AP binding to sections.

Cell culture. DRGs were dissected from E6 or E7 chick embryos and retinas from E6 embryos. Explants were plated onto $10 \mathrm{~mm}$ coverslips coated with laminin $(40 \mu \mathrm{g} / \mathrm{ml})$ and cultured essentially as described by Luo et al. (1993) for 18-24 hr. Olfactory epithelia were isolated from stage 27-29 chick embryos. Tissues containing the nasal sac were dissected out and incubated in Hank's buffer containing $2 \mathrm{mg} / \mathrm{ml}$ Dispase II for $30-60 \mathrm{~min}$ at $20^{\circ} \mathrm{C}$. The olfactory epithelium was isolated with the aid of 30 gauge needles. Care was taken to obtain the whole epithelium with the olfactory nerve attached. The portion of the olfactory epithelium near the olfactory nerve was explanted. Explants were cultured on laminin for $2 \mathrm{~d}$ in the presence of $16 \mu \mathrm{M}$ AraC to suppress the proliferation of non-neuronal cells. Trigeminal (Vth), vestibular (VIIIth), and jugular (proximal Xth) ganglia were dissected from E6 (stage 29-30) chick embryos. Each ganglion was halved, plated onto laminin-coated glass, and cultured for $20 \mathrm{hr}$. The ophthalmic lobe of the trigeminal ganglion was used for all experiments in the present study. The culture medium used was F-12 (Life Technologies) supplemented with $200 \mu \mathrm{g} / \mathrm{ml}$ bovine pituitary extract (Tsao et al., 1982) dialyzed overnight against F-12 medium, 14 mм $\mathrm{NaHCO}_{3}, 2 \mathrm{~mm}$ glutamine, 100 $\mathrm{U} / \mathrm{ml}$ penicillin, $100 \mu \mathrm{g} / \mathrm{ml}$ streptomycin, $6 \mathrm{mg} / \mathrm{ml}$ glucose, $5 \mu \mathrm{g} / \mathrm{ml}$ insulin, $5 \mu \mathrm{g} / \mathrm{ml}$ transferrin, $5 \mathrm{ng} / \mathrm{ml}$ selenious acid, $100 \mu \mathrm{M}$ putrescine, 20 $\mathrm{nm}$ progesterone, and $20 \mathrm{ng} / \mathrm{ml} 7 \mathrm{~S} \mathrm{NGF}$. VIIIth vestibular ganglia were cultured in the same medium supplemented with $10 \mathrm{ng} / \mathrm{ml}$ NT-4, 10 $\mathrm{ng} / \mathrm{ml} \mathrm{BDNF}$, and $8 \mu \mathrm{M}$ AraC; and olfactory epithelium were in the same medium plus $16 \mu \mathrm{M}$ AraC. For the quantitative binding assays to sympathetic neurons, sympathetic ganglionic chains were dissected from E8 chick embryos, incubated for $15 \mathrm{~min}$ in Hank's buffer containing $0.05 \%$ trypsin and $0.5 \mathrm{~mm}$ EDTA at $37^{\circ} \mathrm{C}$, and dissociated into single cells by trituration. Approximately $5 \times 10^{4}$ cells were plated into each polylysine and laminin-coated well in a 48 well plate and were cultured overnight.

Collapse assay. The procedure for the collapse assay was essentially the same as that described previously (Raper and Kapfhammer, 1990). Briefly, $10 \mu \mathrm{l}$ aliquots of diluted recombinant collapsin-1 were added to $500 \mu \mathrm{l}$ of culture medium. The added material was gently mixed into the culture medium, and the cultures were incubated at $37^{\circ} \mathrm{C}$ in $5 \% \mathrm{CO}_{2}$ for $1 \mathrm{hr}$. Cultures were then fixed for $1 \mathrm{hr}$ by gently adding $4 \%$ paraformaldehyde in PBS containing 10\% sucrose. Growth cones without lamellipodia or filopodia were scored as collapsed.

Large-scale production of collapsin-1 for competition experiments. Hi5 insect cells (Invitrogen) were grown in a spinner flask as a suspension culture. Cells were maintained at a density between $10^{5}$ and $10^{6}$ cells $/ \mathrm{ml}$ in EX CELL 405 at $27^{\circ} \mathrm{C}$. The spinners rotated at $100-110 \mathrm{rpm}$. Hi5 cells were infected with baculovirus carrying a recombinant collapsin-1-myc construct (Shepherd et al., 1997) at a density of $10^{6}$ cells $/ \mathrm{ml}$. After $48 \mathrm{hr}$, the supernatant was harvested by gently pelleting the cells. The supernatant was centrifuged at $100,000 \times g$ for $1 \mathrm{hr}$. The resulting pellet was solubilized in $50 \mathrm{~mm}$ Tris, pH 7.4, 0.1\% 3-[(3-cholamidopropyl)dimethylammonio]-1propanesulfonate (CHAPS), and $1 \mathrm{M} \mathrm{NaCl}$ (buffer A) and was centrifuged again at $100,000 \times g$ for $1 \mathrm{hr}$. The supernatant was collected and mixed with $50 \mathrm{~mm}$ Tris, $\mathrm{pH} \mathrm{7.4,0.1 \%} \mathrm{CHAPS,} \mathrm{and} 0.1 \mathrm{M} \mathrm{NaCl}$ (buffer B) at the ratio of 1:5 and was loaded on an S-Sepharose column. The column was washed with buffer B, and pure collapsin-1 was eluted with buffer A.

\section{RESULTS}

\section{Collapsin-1 alkaline phosphatase fusion construct}

Recombinant fusion proteins consisting of human placental alkaline phosphatase and the full coding sequence of collapsin-1 were produced in transiently transfected human $293 \mathrm{~T}$ cells. Two different chimeras were made (Fig. $1 A$ ), one with alkaline phosphatase fused to the $\mathrm{N}$-terminal end of collapsin- 1 behind a signal sequence (AP-Col-1) and another with the enzyme fused to the $\mathrm{C}$-terminal end (Col-1-AP). To verify that collapsin- 1 function is not compromised by the addition of alkaline phosphatase, we tested these fusion proteins for sensory growth cone collapsing activity in an in vitro assay. The specific collapsing activities of both the AP-Col-1 and Col-1-AP fusion proteins are as high or higher than that of collapsin-1 itself (Fig. 1B).

\section{Visualization of collapsin-1 binding on cultured explants}

Collapsin-1 is known to induce the collapse of DRG but not of retinal growth cones in vitro (Luo et al., 1993). A plausible inference is that DRG growth cones will bind collapsin-1, whereas retinal axons may not. Axons extending from E7 DRG explants were incubated with Col-1-AP for $1 \mathrm{hr}$ at $37^{\circ} \mathrm{C}$ and fixed. The presence of alkaline phospatase reaction product demonstrates the binding of collapsin-1 to DRG axons (Fig. $2 B$ ). Not only axons, but also collapsed growth cones, are intensely labeled with Col-1-AP (Fig. 2D). No binding is observed in the explants themselves or in migrating non-neuronal cells. We compared the collapsing activity and intensity of the AP signal at various concentrations of the Col-1-AP probe. If a CU is defined as the concentration of collapsin-1 required to collapse $50 \%$ of DRG growth cones, the binding of Col-1-AP to DRG axons is first detected at $5 \mathrm{CU}$, although staining intensity at this concentration is weak (data not shown). Fifty collapsing units, or $\sim 100$ $\mathrm{ng} / \mathrm{ml}$, give a strong AP signal. To test the specificity of the Col-1-AP signal, we tested whether unlabeled collapsin-1 could compete with Col-1-AP binding. No Col-1-AP binding is detected in DRG cultures simultaneously exposed to a 200 -fold excess $(\sim 20 \mu \mathrm{g} / \mathrm{ml})$ of unlabeled collapsin-1 (Fig. $2 E)$. Even a 20 -fold excess of unlabeled collapsin-1 reduces the intensity of the staining significantly (data not shown). The specificity of Col1-AP binding was confirmed further by testing its ability to bind retinal growth cones. No detectable AP reaction product is observed on retinal ganglion cell axons or growth cones incubated in concentrations of Col-1-AP that give strong DRG labeling (Fig.

\section{$\leftarrow$}

demonstrates expression in the vicinity of the nerve root and surrounding the central spinal trigeminal tract. $D$, A similar pattern of collapsin-1 mRNA expression is seen at the VIIth nerve root (VIIr) and surrounding the central spinal trigeminal $(S p T)$ and the VIIth and VIIIth sensory projections (arrowhead) in the hindbrain. E, AP-Col-1 is expressed in VIIth nerve axons (arrow) and in two central tracts near the entry of VIIth and VIIIth nerve fibers into the hindbrain. $F$, The same section probed for neurofilament allows the visualization of VIIIth nerve axons leaving the VIIIth (vestibular) ganglion on the wall of the otic vesicle $(o v)$ and additional central tracts. $G$, Collapsin- 1 mRNA is expressed strongly in the otic vesicle and in the VIIIth ganglion but not in the VIIth ganglion. $H$, Axons in the vagus nerve label with AP-Col-1 as they enter the hindbrain. I, Additional central tracts within the hindbrain are labeled with anti-neurofilament. $X$, vagus nerve. $J$, Applying the lipophilic axonal tracer DiI to the Vth $(V r)$ and to the VIIth and VIIIth (VII/VIIIr) nerve roots of a hindbrain whole mount (anterior is to the top, and lateral is to the left) labels the more medial spinal trigeminal tract and the more lateral central projections of the VIIth and VIIIth ganglia. VIIth nerve motor neurons are labeled in the lower right. Scale bar, $200 \mu \mathrm{m}$. 
A
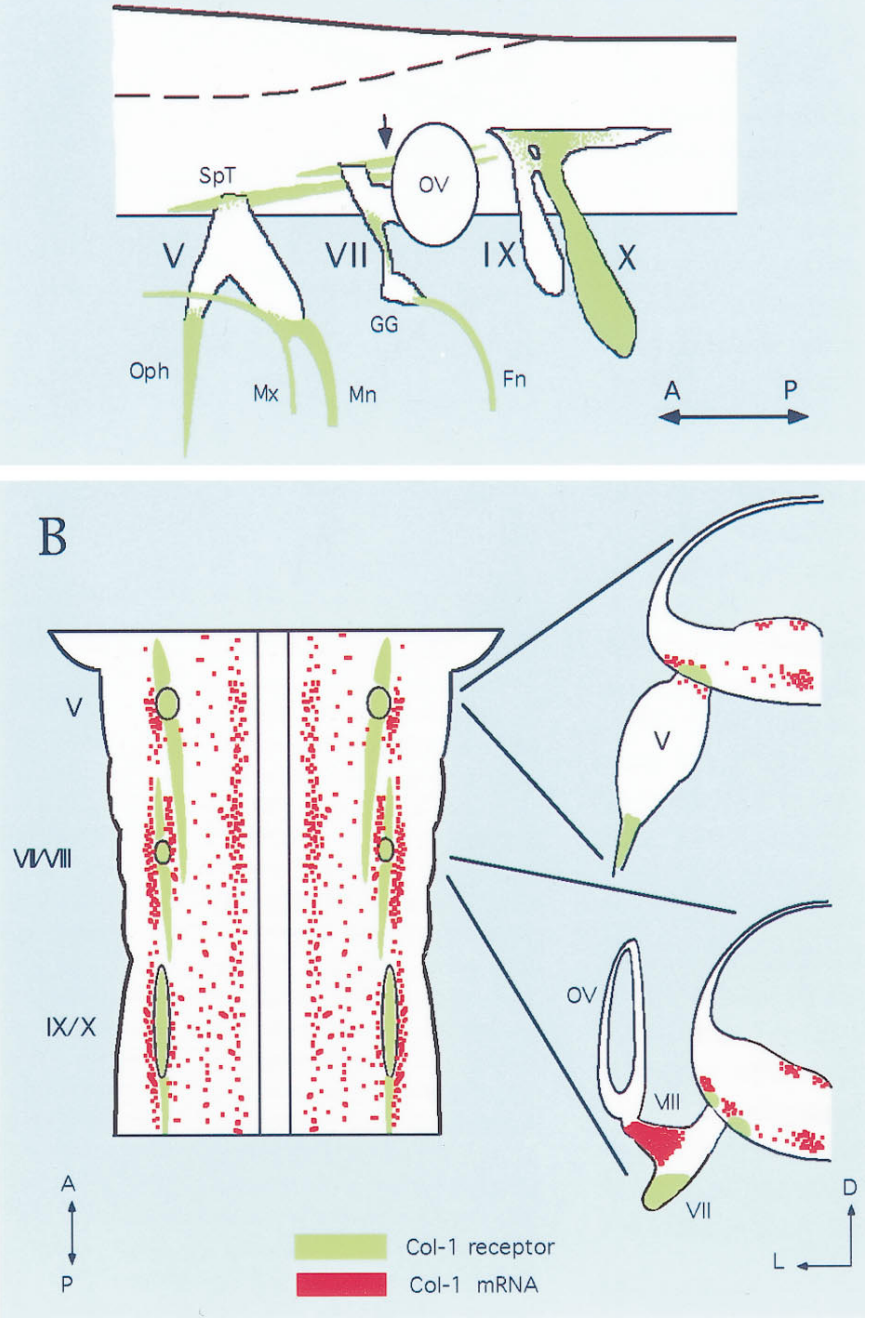

Figure 6. Schematic diagram of AP-Col-1-labeled axons in a stage 25 hindbrain. $A$, Schematic diagram showing the AP-Col-1-labeled axon tract in green. In the hindbrain, the unidentified tract that seems to originate from the VIIth ganglion is labeled (arrow). The most posterior end of this tract has not been followed in the present study. Therefore, only the staining around the VIIth and VIIIth nerve root is shown. Similarly, peripheral projections of IXth (petrosal) and Xth (nodose) ganglia have not been followed. Therefore, distal projections from these ganglia are also omitted. Central descending tracts from the IXth and Xth ganglion were also labeled with AP-Col-1, although it is not shown here. This diagram was made from the reconstruction of $30 \mu \mathrm{m}$ serial sections. $B$, Schematic drawings showing the relationship between AP-Col-1-labeled axons and the distribution of collapsin-1 mRNA in the hindbrain. Left is a schematic of hindbrain viewed from its ventral side. For clarity, only collapsin-1 expression at the ventral side is shown, and the expression in the ventricular side is not shown. AP-Col-1-labeled axons are indicated in green, whereas collapsin-1 mRNA is red. Right are transverse sections at the level of the Vth and of the VIIth and VIIIth nerve roots. V, Trigeminal ganglion; VII, proximal VIIth ganglion; VIII, VIIIth ganglion; $I X$, IXth nerve; $X$, Xth nerve; $F n$, facial nerve; $G G$, geniculate ganglion; $M n$, mandibular nerve; $M x$, maxillary nerve; $O p h$, ophthalmic nerve; $O V$, otic vesicle; $S p T$, spinotrigeminal tract; $A$, anterior; $P$, posterior; $D$, dorsal; $L$, lateral.

$2 F$ ). These results demonstrate that Col-1-AP specifically binds collapsin-1 sensitive axons.

The binding affinity of collapsin-1 to its putative receptors was estimated by examining the binding to dissociated neurons from
E8 sympathetic ganglia. Sympathetic ganglia were chosen for this study because they are responsive to collapsin-1, are almost free from non-neuronal cells, and contain a relatively homogeneous population of neurons. Binding studies (like those just described for sensory neurons) indicate that AP-Col-1 stains the surface of sympathetic axons and cell bodies but not the surface of nonneuronal cells. A 100-fold excess of unlabeled recombinant collapsin-1 prevents AP-Col-1 from binding, demonstrating the specificity of the binding interaction (data not shown). AP-Col-1 binds to dissociated sympathetic neurons in a nearly saturable manner with a $K_{\mathrm{d}}$ of $\sim 800 \mathrm{pm}$ (Fig. $1 C$ ). More-detailed analysis at lower concentrations of the ligand shows that the binding can be fitted well with a two site ligand-receptor binding model (Fig. $1 D)$. A nonlinear regression analysis of the binding data suggests the presence of separate high and low affinity binding sites. The $K_{\mathrm{d}}$ for the high affinity site is estimated to be $\sim 30 \mathrm{pm}$. A Scatchard analysis of the data is consistent with the presence of a high affinity binding site (Fig. $1 D$, inset). In six out of seven cases, Scatchard analysis showed the presence of two binding sites. The number of high affinity sites per sympathetic neuron is estimated to be on the order of $\sim 10^{4}$ sites/cell.

\section{Visualization of collapsin-1 binding in sections}

We next turned our attention to the distribution of collapsin-1 binding in the developing nervous system of the chick. Freshfrozen sectioned embryos were lightly post-fixed in cold methanol and reacted with either Col-1-AP or AP-Col-1, and the alkaline phosphatase was reacted to form an insoluble product that accumulated where the probe was bound. Both probes revealed the same distribution of collapsin-1 binding on sections. Most figures in this paper were stained with AP-Col-1.

\section{Spinal cord}

As would be predicted by their responsiveness to collapsin-1 and their ability to bind Col-1-AP in culture, sensory afferents in the spinal cord are heavily labeled with the probe. In sections of stage 26 embryos, the nascent dorsal sensory columns are well labeled (Fig. 3A). Decreased labeling is obtained when a 50-fold excess of unlabeled collapsin- 1 competes with the AP-Col-1 probe, and no labeling is obtained when a 200-fold excess of unlabeled collapsin-1 is used (Fig. $3 B$ ). Not only the central projections but also the peripheral sensory projections bind AP-Col-1. The sensory nerve just distal to the DRG binds AP-Col-1 before it joins the ventral roots (Fig. $3 A, C$ ). Distal sensory projections may account for AP-Col-1 binding in the peripheral nerves; however, it is possible that binding to motor axons contributes as well. The growth cones of putative ventral spinal cord motor neurons have been shown to collapse in response to collapsin-1 (Shepherd et al., 1996), and the ventral roots of stage 21 embryos are clearly labeled with the probe (Fig. 3D). Much weaker labeling is sometimes apparent in the motor columns and ventral roots of older embryos (Fig. 3A). Collapsin-1 has been shown previously to induce the collapse of cultured sympathetic growth cones. It is therefore not surprising that dissociated sympathetic neurons and axons bind AP-Col-1 and that AP-Col-1 labels sympathetic ganglia in situ (Fig. 3D).

Centrally projecting sensory axons continue to bind AP-Col-1 as development proceeds. The binding of AP-Col- 1 to sensory axons is compared to the distribution of collapsin-1 expressing cells at stage 30 in Figure 4, $A$ and $B$. Collapsin-1 expression is concentrated in the ventral and medial portions of the gray matter, whereas the collapsin- 1 sensitive sensory axons are con- 
centrated in the dorsal and lateral portion of the white matter. This same complementariness is observed later in development. At stage 38, sensory afferents that bind AP-Col-1 fill the dorsal horn (Fig. $4 C$ ) but do not enter the ventral gray matter where collapsin-1 expression is high (Fig. 4D). Interestingly, the most medial portion of the dorsal columns that contains a greater proportion of muscle afferents as compared with cutaneous afferents is less heavily labeled by $\mathrm{AP}-\mathrm{Col}-1$ than are the lateral dorsal columns.

The binding of AP-Col-1 to spinal sensory, motor, and sympathetic axons matches their known sensitivity to collapsin-1 in vitro. However, the real utility of the alkaline phosphatase fusion construct is the identification of new axon tracts likely to be sensitive to collapsin-1. We next examined the hindbrain to determine what neuronal types are likely to be collapsin-1 responsive.

\section{Trigeminal ganglion}

AP-Col-1 binding is first detected in trigeminal axons at stage 18 . The binding pattern in and around the trigeminal ganglion at stage 25 is shown in Figure $5 A$. Figure $6 A$ is a schematic diagram of AP-Col-1 binding reconstructed from serial sections. All three sensory nerves originating in the trigeminal ganglion, the ophthalmic, the maxillary, and the mandibular nerves, are intensely stained with AP-Col-1. The trigeminal root entering the brainstem as well as ascending and descending trigeminal axon tracts in the brainstem are strongly labeled. No AP-Col-1 binding is detected in the trigeminal ganglion itself. Antibodies raised against neurofilaments visualize fibers of passage through the trigeminal ganglion, axon tracts in the brainstem, and the peripheral nerve branches (Fig. 5B). Axons coursing through the ganglion that are not stained with AP-Col-1 are likely to originate within the hindbrain from the motor and mesencephalic sensory nuclei of $\mathrm{V}$. Figures $5 C$ and $6 B$ show the position of collapsin- 1 expression within the hindbrain. Collapsin- 1 is strongly expressed at the lateral border and more weakly expressed at the dorsal and medial margins of the descending trigeminal tract. Surprisingly, collapsin-1 is also expressed in and around the nerve $\mathrm{V}$ root where it enters the brainstem.

\section{Facial, acoustic, and vestibular ganglia}

AP-Col-1 labeling in the vicinity of the fused VIIth (facial) and VIIIth (vestibuloacoustic) ganglia at stage 25 is shown in Figure $5, E$ and $F$. A schematic diagram of AP-Col-1 binding is shown in Figure $6 A$. Weak labeling is detected in the most distal margin of the proximal VIIth ganglion (Fig. $5 E$, arrow). This axonal staining can be followed into the nerve joining the proximal to the distal VIIth (geniculate) ganglion (Fig. 6A). The distal facial nerve also binds the AP-Col-1 probe (Fig. $6 A$ ). There are no detectable AP-Col-1-labeled axons in either the acoustic or the vestibular portions of the VIIIth ganglion. In contrast, collapsin-1 expression is high in both portions of the VIIIth ganglion (Fig. $5 G)$. Collapsin-1 is not expressed in the VIIth ganglion. In the hindbrain, AP-Col-1 labeling is detected in a tract just lateral to the descending spinotrigeminal tract (Fig. $5 E$, arrowhead). As seen in Figure $6 A$, this tract courses just lateral to the entry point for VIIth and VIIIth ganglion axons. Labeling the central projections of VIIth and VIIIth sensory axons by applying DiI to the nerve root indicates that the AP-Col-1-labeled tract corresponds with ascending and descending sensory axons originating in the VIIth and/or VIIIth ganglion (Fig. 5J). Because AP-Col-1 labeling in the periphery is only associated with nerve VII fibers, we attribute the lateral AP-Col-1 positive projection in the brainstem to VIIth ganglion sensory fibers. Figures $5 D$ and $6 B$ show the position of collapsin-1 expression in the hindbrain near the VIIth and VIIIth nerve root. Collapsin-1 is expressed at the lateral border of the facial sensory tract and between the spinal trigeminal and the facial tracts.

\section{Glossopharyngeal and vagus nerves}

AP-Col-1 labeling in the vicinity of the IXth (glossopharyngeal) and $\mathrm{Xth}$ (vagus) nerves at stage 25 is shown in Figure 5, $H$ and $I$. A schematic diagram of AP-Col-1 binding is shown in Figure $6 A$. Strong labeling is seen in the Xth nerve, but no labeling is detected in the IXth nerve or in the distal IXth (petrosal) ganglion. However, the proximal IXth ganglion (superior) is labeled with AP-Col-1 (Fig. 6A).

\section{Olfactory bulb}

One dramatic example of the identification of a new axon tract likely to be sensitive to collapsin-1 is the labeling of axons originating in the olfactory sensory epithelium. The olfactory nerve is strongly stained with AP-Col-1 at stages 23-25 (Fig. 7A). At this stage of development, the olfactory nerve spans the distance between the olfactory epithelium and the telencephalic vesicle. The olfactory bulb has not yet formed, and the sensory axons do not penetrate into the CNS. AP-Col-1 binding is detected along the entire length of the nerve, from the olfactory epithelium to the end of the nerve at the surface of the telencephalon (Fig. 7C). Collapsin-1 is expressed in the olfactory epithelium (Fig. 7B) and in the most superficial layers of the telencephalon (Fig. 7D). Between stages 25 and 30, olfactory sensory axon endings appear to accumulate on the surface of the telencephalon (Fig. 7E), and collapsin-1 continues to be expressed superficially in the telencephalon (Fig. $7 F$ ). Olfactory sensory axons have invaded the nascent olfactory bulb by stage 38 and terminate in a superficial layer. They continue to bind APCol-1 (Fig. 7G). At the same time, collapsin-1 is expressed in what is now an intermediate cell layer between the ventricular zone and the terminating sensory axons (Fig. $7 H$ ).

\section{Responsiveness of cranial sensory and olfactory growth cones to collapsin-1}

To test whether AP-Col-1 binding predicts collapsin-1 responsiveness, we conducted collapse assays on selected axons found in this study to bind AP-Col-1 in situ. Growth cones of axons growing from explanted stage 27-29 olfactory epithelia collapse in response to concentrations of recombinant collapsin-1 comparable with those that affect spinal sensory growth cones (Figs. $8 A, B, 9)$. The same is true for the growth cones of explanted stage 29-30 trigeminal (Vth) ganglia (Figs. 8C,D, 9). Growth cones extending from the geniculate (distal VIIth) and the superior IXth ganglia are found to collapse in response to collapsin-1 (data not shown). Growth cones extending from stage 29-30 jugular (Xth) ganglia also collapse in response to collapsin-1 (Figs. 8G,H, 9). Axons from the VIIIth (vestibuloacoustic) ganglion are not labeled with AP-Col-1 on sections. In agreement with this observation, growth cones of axons originating in explanted stage 29-30 VIIIth vestibular ganglia do not collapse in response to collapsin-1 (Figs. 8E,F, 9). This is true even when they are exposed to a concentration of collapsin-1 10-fold greater than the concentration that induces $50 \%$ collapse of spinal sensory growth cones. All of these activity profiles are as predicted by the pattern of AP-Col-1 binding observed in sections. 

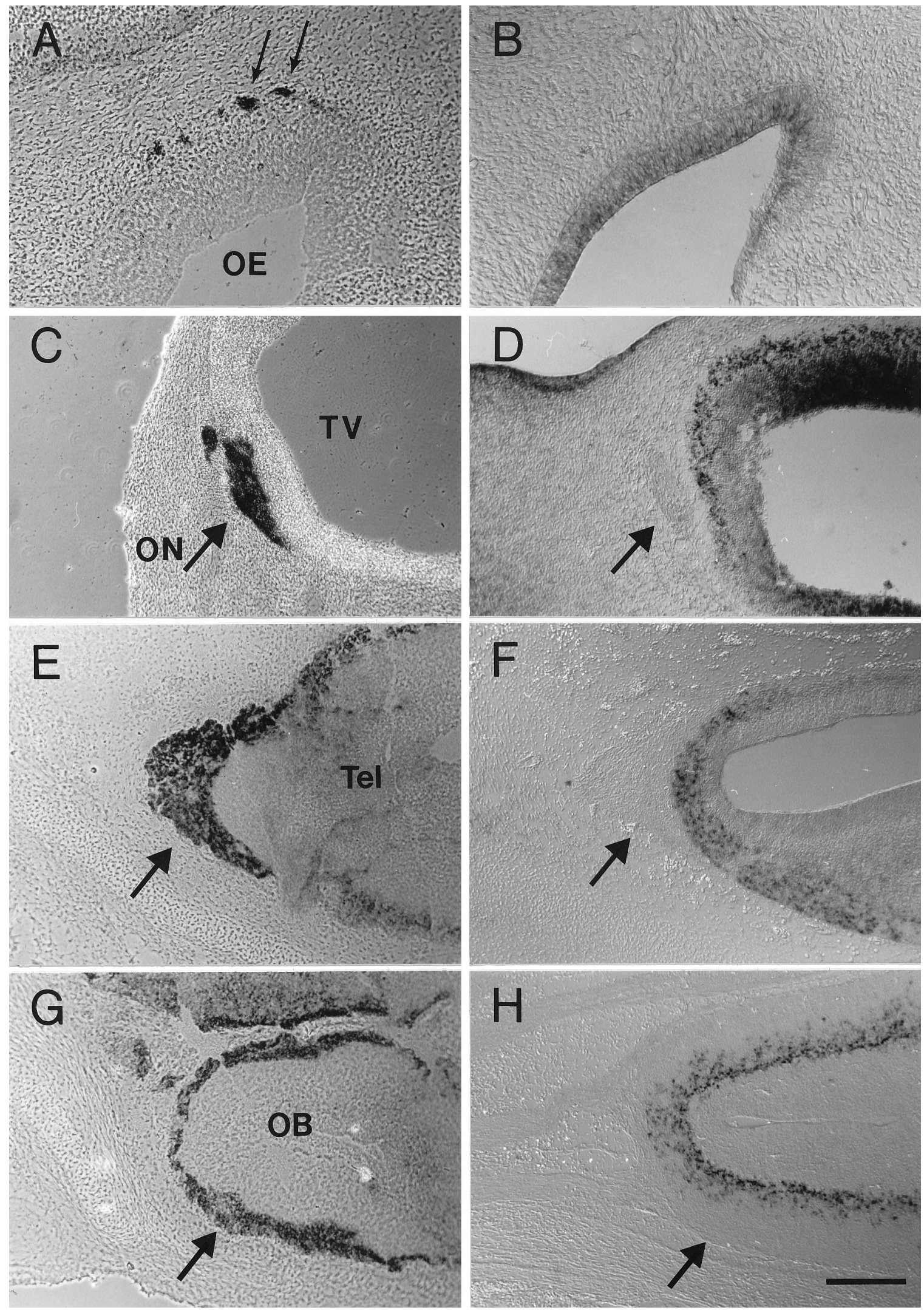

Figure 7. A comparison of AP-Col-1 labeling and collapsin-1 mRNA expression in the developing olfactory system. $A, C, E, G$, Sections probed with AP-Col-1. $B, D, F, H$, In situ hybridizations for collapsin-1 mRNA in matched but separate embryos. $A$, Axons (arrow) leaving the olfactory epithelium $(O E)$ label with AP-Col-1 in stage 25 embryos. $B$, Collapsin-1 mRNA is expressed in the olfactory epithelium at this same stage. $C$, AP-Col-1 labels axons of the olfactory nerve $(O N)$ where they make contact with the rostral tip of the telencephalic vesicle $(T V)$ in a stage 25 embryo. $D$, Collapsin-1 mRNA is expressed in a superficial layer of the telencephalic vesicle. The olfactory nerve is marked with an arrow. Sections (Figure legend continues) 

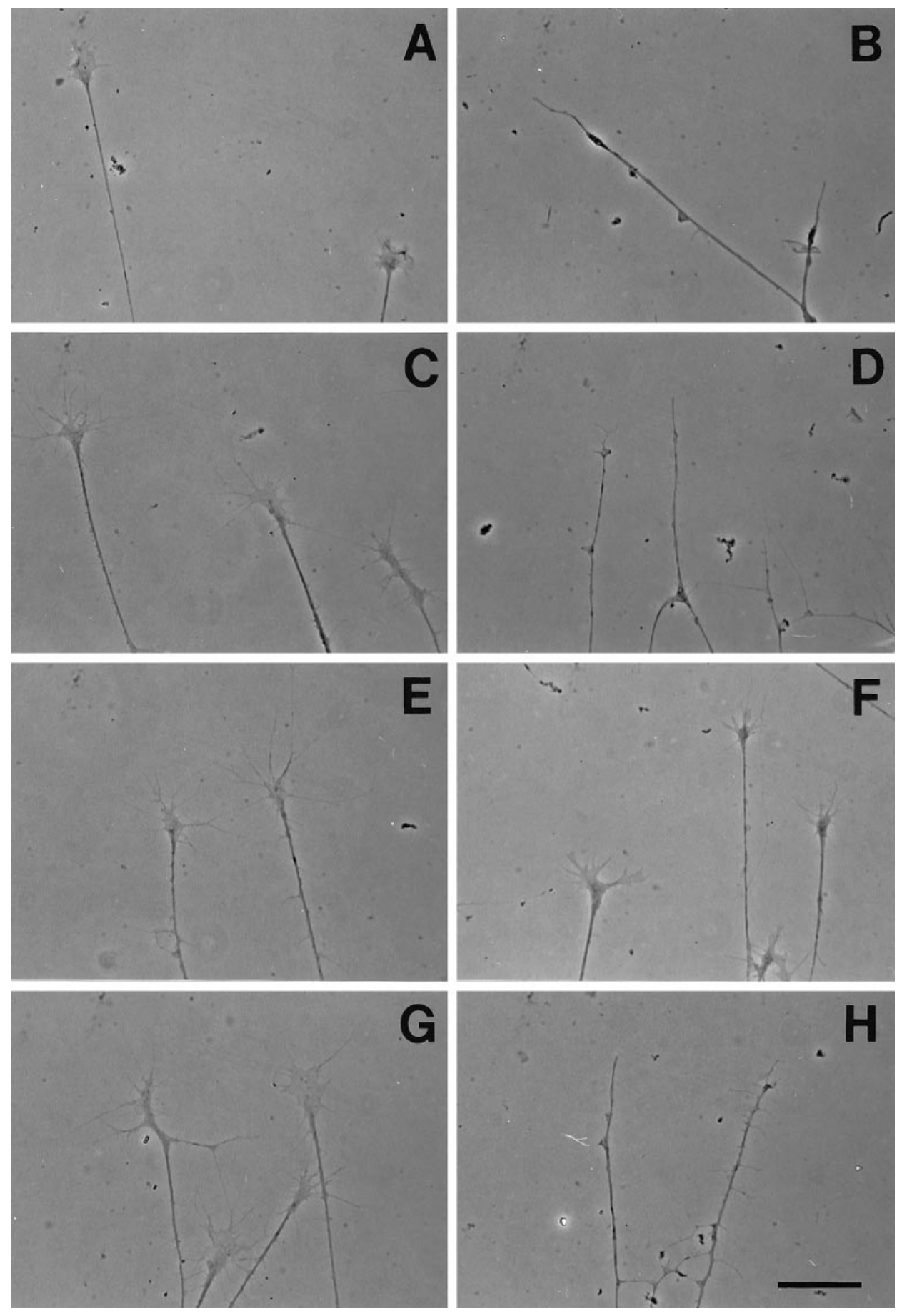

Figure 8. Differential responsiveness of cranial ganglion growth cones to collapsin-1. The morphology of selected growth cones before $(A, C, E, G)$ and after $(B, D, F, H)$ the addition of recombinant collapsin-1. $A, B$, Olfactory growth cones from explanted stage 27-29 olfactory epithelium. $C, D$, Vth (trigeminal) growth cones from stage $30 \mathrm{em}$ bryos. $E, F$, VIIIth (vestibular) growth cones from stage 30 embryos. $G, H$, Proximal Xth (jugular) growth cones from stage $30 \mathrm{em}$ bryos. All cultures were treated with $3 \mathrm{CU}$ of recombinant collapsin-1. Scale bar, $30 \mu \mathrm{m}$.

\section{DISCUSSION}

The objective of this study was to systematically identify axons in the developing chicken nervous system likely to be responsive to the signaling protein collapsin-1. We constructed alkaline phosphatase-collapsin-1 fusion proteins that should bind collapsin-1 receptors and thereby reveal their distribution in tissue sections.

The specificity and utility of these probes is demonstrated by several observations. AP-Col-1 and Col-1-AP are both at least as active as collapsin-1 in an in vitro collapse assay using DRG

$\leftarrow$

were cut coronally, with dorsal right and medial down. E, Olfactory nerve axons (arrow) continue to accumulate on the surface of the telencephalon through stage 31. F, Collapsin-1 continues to be expressed in the most superficial layer of the telencephalon at stage 31 . $G$, Olfactory nerve axons have entered the developing olfactory bulb $(O B)$ and occupy the most superficial layer (arrow) by stage 38. $H$, Collapsin-1 mRNA is expressed at this time in more intermediate layers. Sections in $E-H$ were cut $45^{\circ}$ to the horizontal plane. Rostral is left, and medial is up. Scale bars: $B, 100 \mu \mathrm{m} ; A, C-H, 200 \mu \mathrm{m}$. 


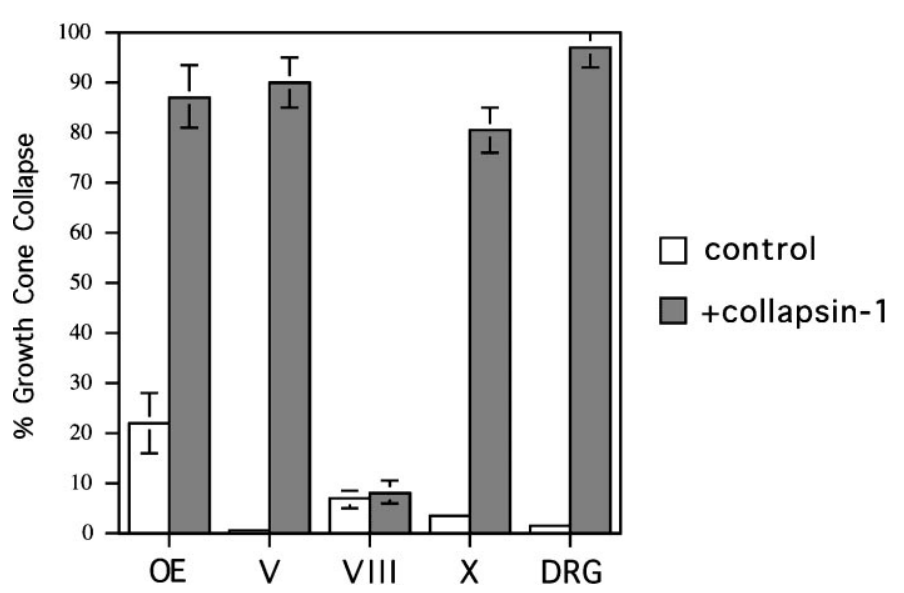

Figure 9. The relative responsiveness of cranial ganglion growth cones to collapsin-1 in vitro. The percentage of collapsed growth cones before (open bars) and after (closed bars) exposure to $10 \mathrm{CU}$ of recombinant collapsin-1. Growth cones extending from explanted olfactory epithelium $(O E)$, the ophthalmic lobe of Vth (trigeminal) ganglia $(V)$, the Xth (jugular) ganglia $(X)$, and DRG ganglia $(D R G)$ are all collapsed in response to exposure to collapsin-1. Growth cones extending from the VIIIth (vestibular) ganglia (VIII) do not collapse in response to collapsin-1. Various neural tissues were cultured as described under Materials and Methods and treated with $10 \mathrm{CU}$ of recombinant collapsin- 1 for $1 \mathrm{hr}$ at $37^{\circ} \mathrm{C}$. After fixation, the growth cones of each explant were scored as having a spread or collapsed morphology. The numbers were collated into the percentage of all counted growth cones having a collapsed morphology. Between 200 and 700 growth cones were scored for each condition.

growth cones, demonstrating their ability to bind the receptor with high affinity. Binding studies of AP-Col-1 on whole cultured sympathetic neurons indicate the presence of a high affinity binding site. This is the predicted result, because sympathetic growth cones collapse in response to concentrations of collapsin-1 of $\sim 30$ pM. We infer that the activation of a high affinity collapsin-1 receptor is likely to initiate sympathetic growth cone collapse.

The ability of AP-collapsin-1 constructs to bind a specific collapsin-1 receptor is supported further by their ability to specifically label appropriate axons in culture. Cultured collapsin-1 responsive DRG axons bind the Col-1-AP probe. Binding of the Col-1-AP probe can be detected with concentrations fivefold greater than those required to obtain $50 \%$ collapse, although concentrations 10-fold greater yield intense staining. Binding of the Col-1-AP probe is competed off with excess unlabeled probe, demonstrating that the probe and collapsin-1 compete for a limited number of specific sites on the cell surface. Cultured retinal axons do not bind detectable Col-1-AP at concentrations that give strong labeling of DRG axons. This result is consistent with the previous observation that retinal axons do not collapse in response to collapsin-1 (Luo et al., 1993). The specificity of the probe is demonstrated further by their specific patterns of binding in sectioned embryos. Only a small number of axon tracts are labeled in sections probed with AP-Col-1 or Col-1-AP. Tracts arising from neurons known to be collapsin-1 responsive are labeled, and those from neurons known to be unresponsive in collapsin-1 in vitro assays are not labeled. This labeling can be competed off with excess unlabeled collapsin-1, again demonstrating that the binding sites are specific and saturable.

The most important test of the utility of the AP-collapsin-1 probes is their ability to identify new collapsin-1 responsive

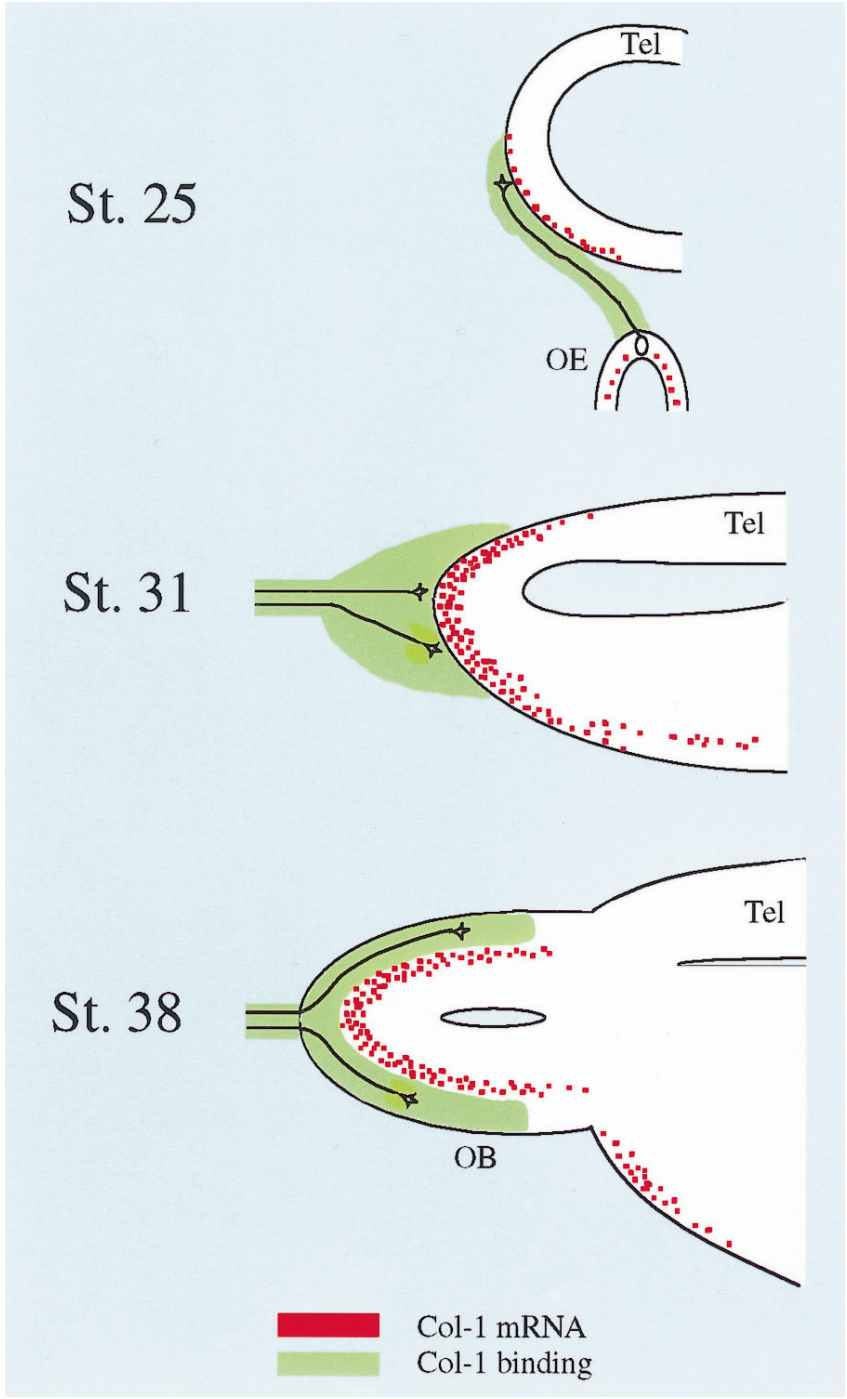

Figure 10. Collapsin-1 is likely first to prevent premature entry of sensory axons into the olfactory bulb and later to confine them to superficial layers. We propose that collapsin- 1 expressed on the surface of the telencephalon prevents the entry of olfactory sensory axons for several days (St. 25, St. 31). Later in development when collapsin-1 expression is restricted to intermediate layers of the olfactory bulb, it could help confine sensory axons and their terminations to the most superficial layers of the bulb. The distribution of collapsin-1 mRNA is shown in red, and that of collapsin-1-binding olfactory axons is in green. A few representative olfactory axons and their growth cones are shown in black. $O E$, Olfactory epithelium; $T e l$, telencephalon; $O B$, olfactory bulb.

axons. The binding of AP-Col-1 and Col-1-AP to primary olfactory sensory axons, as well as to axons extending from the trigeminal, facial, ciliary, and jugular ganglia, implies that these axons possess collapsin- 1 receptors and are likely to be responsive to collapsin-1. Growth cones originating from these explanted neural tissues were subsequently all found to collapse in response to low concentrations of collapsin-1. In contrast, sensory growth cones extending from the VIIIth ganglion do not collapse in response to collapsin-1, as expected by their failure to bind AP-Col-1 in sections.

These findings demonstrate that chimeric alkaline phosphatase-collapsin-1 probes detect a specifically localized collapsin-1 binding component the presence of which on axons correlates with collapsin-1 responsiveness. It is likely that these 
probes visualize a collapsin-1 receptor. Our estimation of affinity constants for the strength of binding between collapsin-1 and its receptor suggests the presence of high affinity receptor on sympathetic axons with a $K_{\mathrm{d}}$ similar to the $30 \mathrm{pm}$ of collapsin- 1 that gives a half maximal collapse response, along with lower affinity sites of $\sim 800$ pM. Presumably, it is this high affinity binding site we see in sections, although we cannot exclude the possibility that we are visualizing a lower affinity binding site that colocalizes with a higher affinity collapsin-1 receptor.

The original rationale for this study was to correlate the distribution of collapsin-1 receptors with the expression pattern of collapsin-1. Such a comparison should suggest possible biological functions for collapsin-1 signaling in vivo. We now turn our attention to the relative distributions of collapsin- 1 and putative collapsin-1 receptors in the spinal cord, brainstem, and olfactory system.

\section{Spinal cord}

Collapsin-1 may play an important role in the organization of DRG sensory afferents in the spinal cord. It is expressed at high levels in the ventral cord and at lower levels in the dorsal gray matter of the cord at stage 23 when sensory afferents first enter the dorsal roots (Shepherd et al., 1996). Sensory axons extend within the developing dorsal columns for the next $2 \mathrm{~d}$, but their afferent branches do not invade the dorsal gray matter until stage 28-29 (Davis et al., 1989). Their entry correlates with a concomitant loss of collapsin-1 expression in the nascent dorsal horn (Shepherd et al., 1997). All growth cones extending in vitro from E7 DRG ganglia appear to collapse in response to collapsin-1 (Luo et al., 1993; Püschel et al., 1996; Sharma et al., 1996; Shepherd et al., 1997). It is therefore possible that the loss of collapsin-1 expressing cells in the dorsal gray matter around stage 29 permits collapsin-1 sensitive sensory afferents to enter the spinal gray matter. Consistent with this hypothesis is the presence of strong AP-Col-1 binding on axons within the dorsal columns throughout the time course of these events.

DRG afferents can be classified by their ultimate destinations in the spinal cord. Most afferents terminate in various laminae within the dorsal horn and never extend into the ventral cord. A minority of afferents, including the group Ia stretch receptors, ultimately extend into and synapse within the ventral cord (Brown, 1981; Willis and Coggeshall, 1991). Collapsin-1 expression is gradually confined to the ventral cord between stages 30 and 36. Dorsally terminating afferents remain collapsin-1 sensitive and are confined to the dorsal cord by ventrally expressed collapsin-1, whereas ventrally terminating afferents become collapsin-1 insensitive and are thereby able to invade the ventral cord (Messersmith et al., 1995; Behar et al., 1996; Püschel et al., 1996; Shepherd et al., 1997). In agreement with this, AP-Col-1 only labels the dorsal gray matter. Interestingly, by stage 38 the most medial portion of the dorsal white matter binds significantly less AP-Col-1 than does the more lateral dorsal white matter (Fig. 4C). This medial portion of the white matter is enriched in TrkC-positive, ventrally invading afferents (Oakley et al., 1997). TrkA-positive dorsally terminating afferents are predominant in the more lateral white matter where strong AP-Col- 1 binding is evident. These results suggest that ventrally invading afferents become collapsin- 1 insensitive because they lose the collapsin-1 receptor. The cell- and time-specific downregulation of collapsin-1 receptor expression may control which sensory afferents invade ventrally and at what developmental time they begin their invasion.

\section{Hindbrain}

Sensory axons extending from ganglia contributing to the $\mathrm{Vth}$ (trigeminal), VIIth (facial), and Xth (vagus) cranial nerves bind AP-Col-1 and collapse in response to collapsin-1. This collapsin-1 responsiveness could play a role in the guidance of these axons in the periphery, as proposed previously for the peripheral axons of DRG sensory axons (Wright et al., 1995). The finding that collapsin-1 responsive sensory axons abut collapsin-1-expressing cells in the brainstem suggests that collapsin-1 may help to define the medial and lateral limits of these central sensory tracts. Collapsin-1 expression between the central spinal trigeminal and VIIth sensory tracts may help keep them separate.

\section{Fused VIIth and VIIIth ganglia}

The VIIth and VIIIth nerves share the same entry point into the hindbrain. In the chick, there are two facial sensory ganglia, a distal (geniculate) ganglion and a more proximal ganglion that is fused with the VIIIth (vestibular) ganglion (Yamamoto and Schwarting, 1991). Axons extending from these ganglia enter the hindbrain through the fused VII and VIII nerve root. Centrally projecting facial sensory axons must therefore navigate through a choice point where they choose to enter the VII and VIII root and not the VIIIth ganglion. Collapsin-1 is expressed in both the vestibular and acoustic parts of the VIIIth ganglion. AP-Col-1 binds to VIIth nerve axons, and growth cones extending from explanted VIIth ganglia respond to collapsin-1. This complementary pattern of collapsin-1 expression and collapsin-1 receptor distribution suggests that collapsin-1 in the VIIIth ganglion denies entry to collapsin-1 responsive VIIth nerve axons growing toward the brainstem.

\section{Olfactory system}

Just as spinal sensory afferents observe a waiting period before they enter the gray matter of the spinal cord, axons in the olfactory nerve wait on the surface of the telencephalon before entering the developing olfactory bulb. The time course of olfactory nerve innervation of the bulb has been extensively studied in rodents (Doucette, 1989; Marin-Padilla and Amieva, 1989; Santacana et al., 1992). Olfactory sensory epithelial axons grow through the mesenchyme between the epithelium and the telencephalon, turn anteriorly once they reach the telencephalon, and stop where the olfactory bulb will later differentiate. Although a small number of pioneer olfactory axons transiently penetrate into the telencephalon, the vast majority of olfactory axons accumulate for days outside the CNS without entering (Valverde et al., 1992; Gong and Shipley, 1995). These fibers only enter the CNS as the telencephalic vesicle begins to evaginate when the olfactory bulb starts to form. Olfactory nerve axons ultimately terminate in glomeruli located within a superficial layer of the bulb.

Although much less information is available about these events in the developing chick, our observations are consistent with this general scheme (Fig. 10). Olfactory nerve axons have arrived at the telencephalic surface by stage 25 (Fig. 10, St. 25) but have not invaded the CNS even by stage 31 (Fig. 10, St. 31). Interestingly, throughout this time period, collapsin-1 is expressed within the most superficial layers of the telencephalic vesicle (for similar observations in the rat, see Giger et al., 1996). Our demonstration that AP-Col-1 binds to olfactory sensory axons and that collapsin-1 collapses olfactory growth cones in vitro suggests that collapsin-1 prevents olfactory axon entrance into the telencephalon during this period. By stage 38 , olfactory axons have invaded 
the nascent olfactory bulb and now occupy its outermost layer (Fig. 10, St. 38). At this stage of development, collapsin-1 is expressed in an intermediate layer just beneath the olfactory axon terminations. We therefore predict that sensory axons are confined to superficial layers of the olfactory bulb by the expression of collapsin-1 in intermediate layers.

Both in the dorsal horn of the spinal cord and in the olfactory bulb, collapsin-1 receptive sensory axons are excluded for several days from immediately adjacent areas that express collapsin-1. In both cases, the final pattern of sensory axon termination is complementary to the distribution of collapsin- 1 expression. Thus, both the timing of target penetration and the final spatial localization of terminations may be controlled by the timing and localization of collapsin-1 expression.

\section{REFERENCES}

Adams RH, Betz H, Püschel AW (1996) A novel class of murine semaphorins with homology to thrombospondin is differentially expressed during early embryogenesis. Mech Dev 57:33-45.

Behar O, Golden JA, Mashimo H, Schoen FJ, Fishman MC (1996) Semaphorin III is needed for normal patterning and growth of nerves, bones and heart. Nature 383:525-528.

Brown AG (1981) Organization of the spinal cord. The anatomy and physiology of identified neurons. New York: Springer.

Chan SS-Y, Zheng H, Su M-W, Wilk R, Killeen MT, Hedgecock EM, Culotti JG (1996) UNC-40, a C. elegans homolog of DCC (deleted in colorectal cancer), is required in motile cells responding to UNC-6 netrin cues. Cell 87:187-195.

Cheng H-J, Nakamoto M, Bergemann AD, Flanagan JG (1995) Complementary gradients in expression and binding of ELF-1 and Mek4 in development of the topographic retinotectal projection map. Cell 82:371-381.

Davis BM, Frank E, Johnson FA, Scott SA (1989) Development of central projections of lumbosacral sensory neurons in the chick. J Comp Neurol 279:556-566.

Desai CJ, Gindhart JG, Goldstein LSB, Zinn K (1996) Receptor tyrosine phosphatases are required for motor axon guidance in the Drosophila embryo. Cell 84:599-609.

Doucette R (1989) Development of the nerve fiber layer in the olfactory bulb of mouse embryos. J Comp Neurol 285:514-527.

Fan J, Raper JA (1995) Localized collapsing cues can steer growth cones without inducing their full collapse. Neuron 14:263-274.

Flanagan JG, Leder P (1990) The kit ligand: a cell surface molecule altered in steel mutant fibroblasts. Cell 63:185-194.

Giger RJ, Wolfer DP, De Wit GMJ, Verhaagen J (1996) Anatomy of rat semaphorin III/collapsin-1 mRNA expression and relationship to developing nerve tracts during neuroembryogenesis. J Comp Neurol 375:378-392.

Gong Q, Shipley MT (1995) Evidence that pioneer olfactory axons regulate telencephalon cell cycle kinetics to induce the formation of the olfactory bulb. Neuron 14:91-101.

Hedgecock EM, Culotti JG, Hall DH (1990) The unc-5, unc-6, and unc-40 genes guide circumferential migrations of pioneer axons and mesodermal cells on the epidermis in C. elegans. Neuron 4:61-85.

Henkemeyer M, Orioli D, Henderson JT, Saxton TM, Roder J, Pawson T, Klein R (1996) Nuk controls pathfinding of commissural axons in the mammalian central nervous system. Cell 86:35-46.

Keino-Masu K, Masu M, Hinck L, Leonardo ED, Chan SS-Y, Culotti JG, Tessier-Lavigne M (1996) Deleted in colorectal cancer (DCC) encodes a netrin receptor. Cell 87:175-185.

Kolodkin AL, Matthes DJ, Goodman CS (1993) The semaphorin genes encode a family of transmembrane and secreted growth cone guidance molecules. Cell 75:1389-1399.

Koppel AM, Feiner L, Kobayashi H, Raper JA (1997) A 70 amino acid region within the semaphorin domain activates specific cellular response of semaphorin family members. Neuron, in press.

Krueger NX, Van Vactor D, Wan HI, Gelbart WM, Goodman CS, Saito H (1996) The transmembrane tyrosine phosphatase DLAR controls motor axon guidance in Drosophila. Cell 84:611-622.
Luo Y, Raible D, Raper JA (1993) Collapsin: a protein in brain that induces the collapse and paralysis of neuronal growth cones. Cell 75:217-227.

Luo Y, Shepherd I, Li J, Renzi MJ, Chang S, Raper JA (1995) A family of molecules related to collapsin in the embryonic chick nervous system. Neuron 14:1-20.

Marin-Padilla M, Amieva MR (1989) Early neurogenesis of the mouse olfactory nerve: golgi and electron microscopic studies. J Comp Neurol 288:339-352.

Messersmith EK, Leonardo ED, Shatz CJ, Tessier-Lavigne M, Goodman CS, Kolodkin AL (1995) Semaphorin III can function as a selective chemorepellent to pattern sensory projections in the spinal cord. Neuron 14:949-959.

Miyazaki J, Takaki S, Araki K, Tashiro F, Tominaga A, Takatsu K, Yamamura K (1989) Expression vector system based on the chicken $\beta$-actin promoter directs efficient production of interleukin-5. Gene 79:269-277.

Nakamoto M, Cheng H-J, Friedman GC, Mclaughlin T, Hansen MJ, Yoon CH, O'Leary DDM, Flanagan JG (1996) Topographically specific effects of ELF-1 on retinal axon guidance in vitro and retinal axon mapping in vivo. Cell 86:755-766.

Oakley RA, Lefcort FB, Clary DO, Reichardt LF, Prevette D, Oppenheim RW, Frank E (1997) Neurotrophin-3 promotes the differentiation of mouse spindle afferents in the absence of peripheral targets. J Neurosci 17:4262-4274.

Püschel AW, Adams RH, Betz H (1995) Murine semaphorin D/collapsin is a member of a diverse gene family and creates domains inhibitory for axonal extension. Neuron 14:941-948.

Püschel AW, Adams RH, Betz H (1996) The sensory innervation of the mouse spinal cord may be patterned by differential expression of and differential responsiveness to semaphorins. Mol Cell Neurosci 7:419-431.

Raper JA, Kapfhammer JP (1990) The enrichment of a neuronal growth cone collapsing activity from embryonic chick brain. Neuron 4:21-29.

Santacana M, Heredia M, Valverde F (1992) Transient pattern of exuberant projections of olfactory axons during development in the rat. Dev Brain Res 70:213-222.

Serafini T, Kennedy TE, Galko MJ, Mirzayan C, Jessell TM, TessierLavigne M (1994) The netrins define a family of axon outgrowthpromoting proteins homologous to C. elegans UNC-6. Cell 78:409-424.

Sharma K, Luo Y, Raper J, Frank E (1996) Collapsin-1 delays the growth of sensory afferents into the dorsal spinal cord. Soc Neurosci Abstr 22:585.6.

Shepherd I, Luo Y, Raper JA, Chang S (1996) The distribution of collapsin-1 mRNA in the developing chick nervous system. Dev Biol 173:185-199.

Shepherd IT, Luo Y, Lefcort F, Reichardt LF, Raper JA (1997) A sensory axon repellent secreted from ventral spinal cord explants is neutralized by antibodies raised against collapsin-1. Development 124:1377-1385.

Tsao MC, Walthall BJ, Ham RG (1982) Clonal growth of normal human epidermal keratinocytes in a defined medium. J Cell Physiol 110:219-229.

Valverde F, Santacana M, Heredia M (1992) Formation of an olfactory glomerulus: morphological aspects of development and organization. Neuroscience 49:255-275.

Willis WD, Coggeshall RE (1991) Sensory mechanisms of the spinal cord. London: Plenum.

Wright DE, White FA, Gerfen RW, Silos-Santiago I, Snider WD (1995) The guidance molecule semaphorin III is expressed in regions of spinal cord and periphery avoided by growing sensory axons. J Comp Neurol 361:321-333.

Yamamoto M, Schwarting G (1991) The formation of axonal pathways in developing cranial nerves. Neurosci Res 11:229-260.

Zhang J-H, Cerretti DP, Yu T, Flanagan JG, Zhou R (1996) Detection of ligands in regions anatomically connected to neurons expressing the Eph receptor Bsk: potential roles in neuron-target interaction. J Neurosci 16:7182-7192. 